\title{
CARBON STARS AT HIGH GALACTIC LATITUDE ${ }^{1}$
}

\author{
GREG BOTHUN \\ University of Oregon, Eugene, Oregon 97403 \\ J. H. Elias \\ Cerro Tololo Inter-American Observatory, National Optical Astronomy Observatories, ${ }^{2}$ P.O. Box 26732, Tucson, Arizona 85726 \\ G. MAcAlpine \\ University of Michigan, Ann Arbor, Michigan 48109-1090
}

\author{
K. Matthews, J. R. Mould, G. Neugebauer, AND I. N. Reid \\ Palomar Observatory, Caltech 105-24, Pasadena, California 91125 \\ Received 5 December 1990; revised 6 February 1991
}

\begin{abstract}
Photometry and kinematics are presented for a sample of objective prism selected carbon stars towards the north and south Galactic poles. Distances are determined by fitting the infrared colors to a giant branch. If these stars are like the carbon stars seen in dwarf spheroidal galaxies, the median distance of the sample is $28 \mathrm{kpc}$. If they are more like the carbon stars found recently in the Galactic bulge, they may be only half as distant. The surface density of carbon stars as a function of distance is remarkably consistent with an $R^{1 / 4}$ density profile for the Galactic halo. This density profile can be traced to $\approx 15$ scale radii and fills a volume similar to that occupied by globular clusters. The data yield an effective radius of either 7.0 or $3.5 \mathrm{kpc}$ depending on choice of distance scale. The velocity dispersion of the sample is $96 \pm 12 \mathrm{~km} / \mathrm{s}$. A kinematic model in which vertical velocity dispersion is independent of height above the Galactic plane seems in best accord with the data.
\end{abstract}

\section{INTRODUCTION}

The formation of asymptotic giant branch carbon stars by the third dredge-up mechanism (Iben 1975, see also Lattanzio 1986; Boothroyd \& Sackman 1988) has found experimental confirmation in several studies of the intermediate age population of the Magellanic Clouds (e.g., Cohen et al. 1981; Mould \& Da Costa 1988). Stars of the Galaxy's first generation of $0.8 \mathscr{M}_{\odot}$ are not thought to experience the third dredge up, however, and the existence of halo carbon stars has been ascribed to mass transfer in binary systems (McClure 1984) or to events connected with the helium flash. Identification of high latitude carbon stars as Population II would help confirm the mechanism proposed by McClure (1984), whereas their identification as intermediate-age carbon stars would lend support to the model of Rodgers \& Paltoglou (1984) in which the Galactic halo has been contaminated by debris from a recent encounter with a Magellanic-type galaxy.

In addition, observations of carbon stars which are located at high Galactic latitude provide insight on the dynamical nature of the Galactic halo. Since very accurate radial velocities can be obtained from carbon star spectra (e.g., Aaronson 1983), they form a set of distant tracers of the gravitational field of the Galaxy. In particular, the vertical velocity

\footnotetext{
${ }^{1}$ Based on observations obtained at Palomar Observatory, ${ }^{2}$ Cerro Tololo Inter-American Observatory and Las Campanas Observatory. ${ }^{2}$

${ }^{2}$ NOAO is operated by AURA, Inc. under contract to NSF. Las Campanas Observatory is owned by the Carnegie Institution of Washington. Palomar Observatory is owned and operated by the California Institute of Technology.
}

dispersion of a sample of carbon stars that are located near the Galactic poles, can constrain models of the halo velocity ellipsoid (Pier 1984; Ratatunga \& Freeman 1985). Morever, the run of carbon star surface density with galactocentric distance may provide a probe of the structure of the Galactic halo to distances at least as large as is offered by either globular clusters or RR Lyrae stars (e.g., Kinman 1991).

While no large-scale survey for halo carbon stars has been undertaken, we note that objective prism surveys designed to detect emission line objects are capable of readily detecting carbon stars because their strong bandheads give them an emission line appearance. Fortunately, two high latitude objective prism samples are readily accessible. The northern sample comes from the Case Low Dispersion Northern Sky Survey (Pesch \& Sanduleak 1988). The southern sample comes from the University of Michigan Thin Prism survey which was carried out at the Curtis-Schmidt telescope (MacAlpine \& Lewis 1978, and references therein).

In this paper we report on photometric and spectroscopic observations of this sample. $J H K$ photometry provides an accurate measure of effective temperature which can be used as a necessary but not sufficient distinction between a Population II carbon star and one of more intermediate age. In addition, this photometry provides an estimate of $\mathscr{M}_{\text {bol }}$ from which approximate distances can be derived. Our goal with these observations is to (1) measure accurate radial velocities to constrain the vertical velocity dispersion at high latitude, (2) establish the surface density falloff in comparison to a de Vaucouleurs $r^{1 / 4}$ law, (3) ascertain if any of these carbon stars are similar to those observed in intermediateage populations in the $\mathrm{LMC}$, and (4) search for differences in carbon star properties between the NGP and SGP samples. 


\section{OBSERVATIONS \\ 2.1 Spectroscopy}

Slit spectra of five of the Case (CLS) stars were obtained by Mould et al. (1984) at Palomar Observatory. In Fig. 1 we show spectra of a further 15 stars obtained with a similar configuration of the Double Spectrograph (Oke \& Gunn
1982). These data were obtained in March 1988. Radial velocities of these stars were measured with respect to the standards HD 57884, 79319, and 156074 (McClure 1984; 1989, private communication), and are recorded in Table 1. Our derived values for the standards agree with the published values of McClure (1984) to an r.m.s. accuracy of $9 \mathrm{~km} \mathrm{~s}^{-1}$. One star, CLS105, was observed to have a radial-velocity
CLS

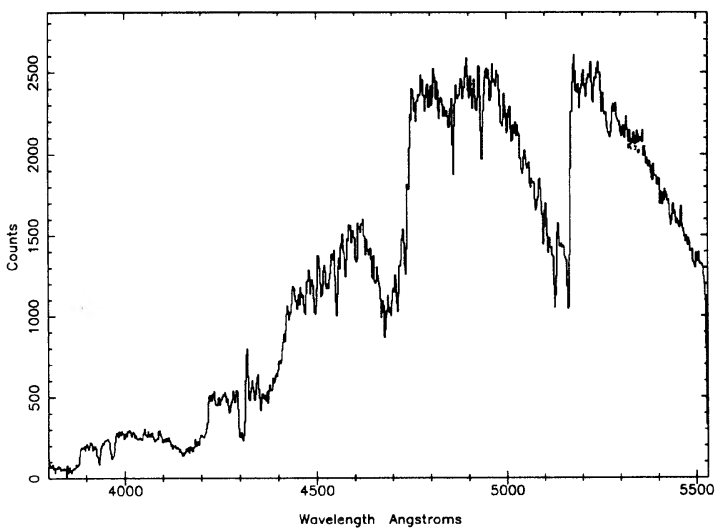

CLS 26

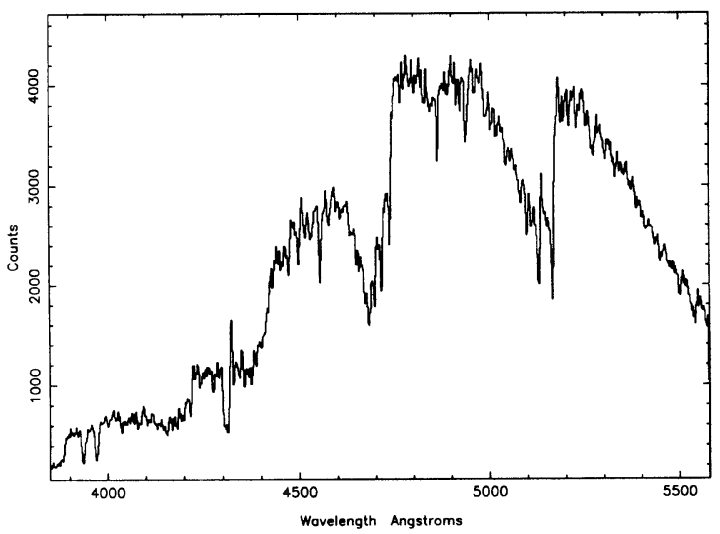

CLS 48

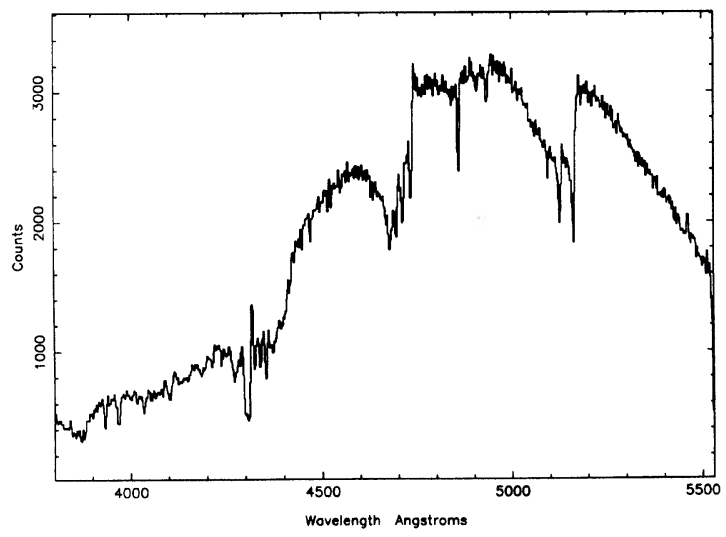

CLS 14

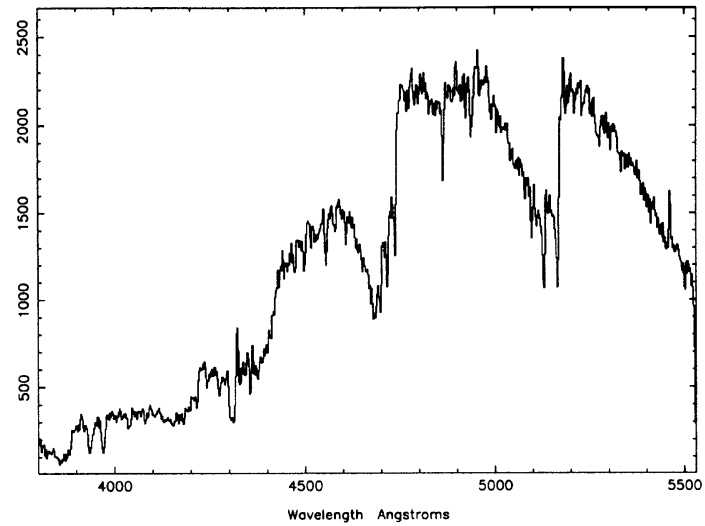

CLS 38

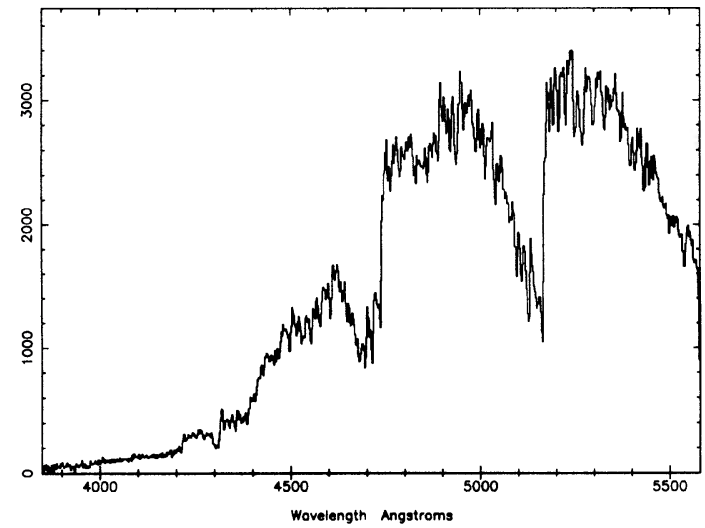

CLS 67

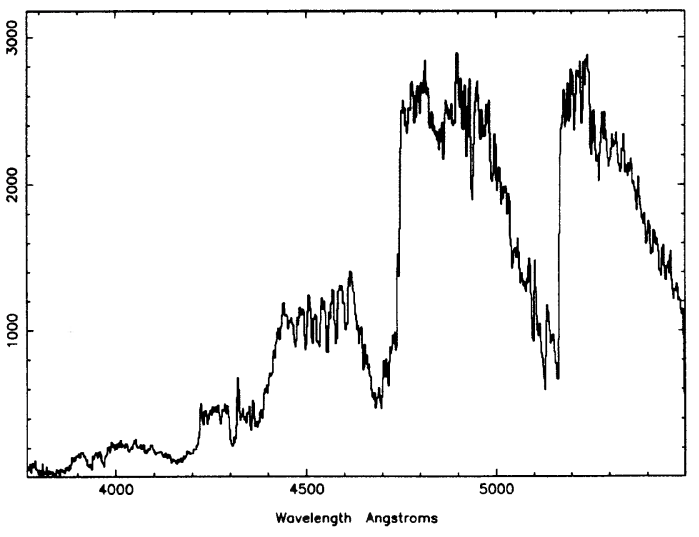

FIG. 1. Palomar spectra of Case carbon stars from Table 1. 

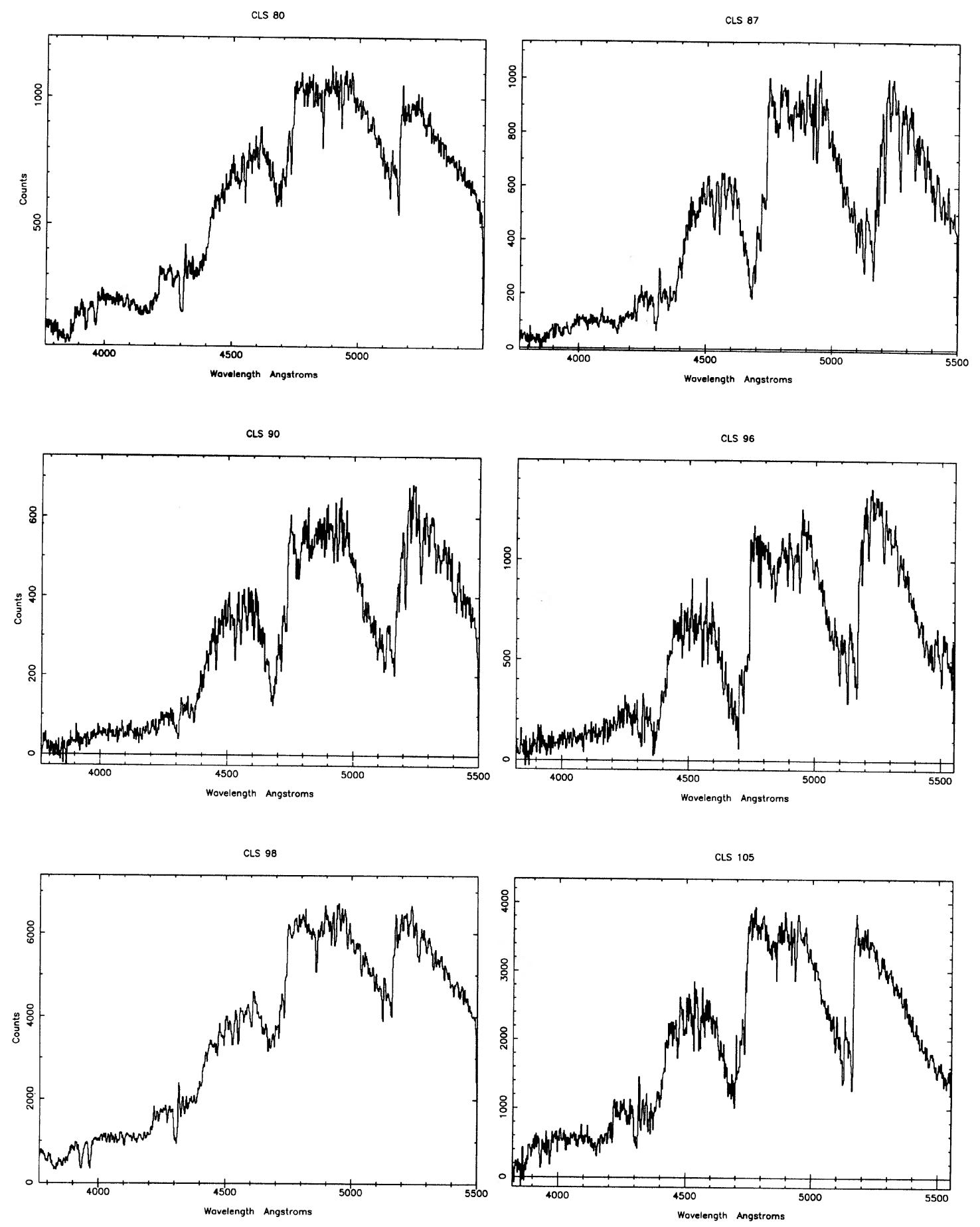

FIG. 1. (continued)

variation of $40 \mathrm{~km} \mathrm{~s}^{-1}$ over two successive nights. Our quoted velocity is an average of these two nights of observations. Another star, CLS23, was observed to have a significantly different velocity from that tabulated by Mould et al. (1984). This was the only star from that earlier sample which was reobserved in 1988. The following stars are not included in Fig. 1 or Table 1, as their spectra proved to be of
K or M type: CLS 1, 3, 12, 20, 21, 75, 78. Our spectra of CLS stars are similar to those shown by Green \& Margon (1990), with the addition that radial velocities are reported here. There are 11 stars in common between this work and that of Green \& Margon (1990).

In the UM survey, carbon star candidates were selected by eye by one of us (G. M.) on the basis of red continuum colors 
TABLE 1. Photometry and kinematics of the NGP sample.

\begin{tabular}{lllll}
\hline \hline CLS & $\begin{array}{l}\mathrm{J} \\
(\mathrm{mag})\end{array}$ & $\begin{array}{l}\mathrm{H} \\
(\mathrm{mag})\end{array}$ & $\begin{array}{l}\mathrm{K} \\
(\mathrm{mag})\end{array}$ & $\begin{array}{l}\text { Velocity } \\
(\mathrm{km} / \mathrm{s})\end{array}$ \\
\hline 9 & 11.20 & 10.69 & 10.59 & -59 \\
14 & 13.45 & 12.95 & 12.90 & 123 \\
$23^{4}$ & 12.33 & 11.83 & 11.72 & 47 \\
26 & 12.15 & 11.71 & 11.62 & 142 \\
31 & 15.49 & 14.97 & 14.69 & -44 \\
38 & 10.03 & 9.36 & 9.15 & -24 \\
48 & 12.14 & 11.73 & 11.65 & -27 \\
67 & 12.19 & 11.61 & 11.38 & 40 \\
80 & 10.69 & 10.27 & 10.17 & -217 \\
87 & 13.46 & 12.89 & 12.70 & -25 \\
90 & 11.85 & 11.21 & 10.97 & -67 \\
96 & 13.75 & 13.14 & 12.82 & 164 \\
98 & 10.67 & 10.16 & 9.99 & -135 \\
105 & 12.43 & 11.98 & 11.83 & -189 \\
112 & 13.28 & 12.92 & 12.79 & -58 \\
\hline
\end{tabular}

${ }^{3}$ Heliocentric radial velocity in $\mathrm{km} / \mathrm{sec}$

${ }^{4}$ Radial velocity significantly different from the previous observation in March 1984.

combined with "emission" features that were more characteristic of molecular band heads than the narrower lines of extragalactic emission line galaxies. As will be noted later, the southern sample is significantly redder than the northern sample, which may reflect this requirement of "red" colors. The UM survey is relatively complete, for stellar objects, to $B=17.5$ mag. In addition, Salzer (1987) discovered one carbon star from the UM survey with $B=20.2 \mathrm{mag}$ and $B-V=3.2 \mathrm{mag}$. Our selection from the Curtis-Schmidt plates was restricted in area, and our criteria yielded a total of 32 carbon star candidates. Spectra of the southern carbon star candidates were obtained in October 1988 using the modular spectrograph (Schechter 1988) on the $2.5 \mathrm{~m}$ du Pont telescope at Las Campanas Observatory. The spectrograph was used in a cross-dispersed echellette configuration, with a 100 lines $\mathrm{mm}^{-1}$ reflection grating and a 200 lines $\mathrm{mm}^{-1}$ transmission grating as a cross-disperser, and the spectra cover the wavelength range $\sim 4700$ to $\sim 9600 \AA$. The detector is an $800 \times 800$ format TI chip, with the $15 \mu \mathrm{m}$ pixels corresponding to $70 \mathrm{~km} \mathrm{~s}^{-1}$. The resolution varies from 1.7 to 2.5 pixels (FWHM) across the chip. Sample spectra are shown in Fig. 2.

Our data reduction methods are described in detail by Reid, et al. (1990). To summarize, the spectra were extracted with the appropriate sky subtraction; wavelengths were calibrated using exposures of helium, neon and argon lamps that bracket the stellar exposure; and spectra were set on a flux scale using observations of LTT 9239 (Stone \& Baldwin 1983). A comparison with other flux standards shows that the final flux calibration is accurate to $\sim 10 \%$. The spectra were also rebinned on a logarithmic wavelength scale and cross-correlated against several carbon stars, including VX Aql, HD 189711, and R CMi. Our observations of stars of known velocity lead to an estimated uncertainty of 19 $\mathrm{km} \mathrm{s}^{-1}$ in the derived velocities.

We obtained spectra of 30 of the 32 stars identified in the Michigan survey (Table 2). Of these 30,13 are carbon stars. Most of the remaining stars are late-type $M$ giants, but two have strong $\mathrm{Na} \mathrm{D}$ lines, indicating that they are $\mathrm{M}$ dwarfs, while a further two stars are K-type stars with strong $\mathrm{H} \alpha$ emission and $\mathrm{Ca} / \mathrm{Fe} \lambda 6498$ absorption. These spectroscopic results indicate that selecting carbon stars via initial inspec- tion of objective prism surveys yields a $50 \%$ success rate. $A$ posteriori inspection of the plates, based on the spectroscopic results, reveals that the carbon stars do have an objective prism signature that is slightly different from late $\mathbf{M}$ stars and thus a more careful search might yield a higher success rate. Better success might also be acheived by use of a narrowband filter system, such as that employed by Cook \& Aaronson (1989) and Green \& Margon (1991). However, we note that the surface density of carbon stars at high latitude is quite low. For the sample at the SGP we derive a surface density of order 1 carbon star per 30 square degrees.

\subsection{Photometry}

Infrared photometry of the northern sample was obtained with the $5 \mathrm{~m}$ telescope at Palomar Observatory with a single channel InSb detector on three photometric nights in 1988 and 1989. Repeat observations of six stars allow us to estimate mean uncertainties of 0.02 mag at $J, H$, and $K$. These measurements, on the CIT/CTIO system (Elias et al. 1982), are recorded in Table 1.

Observations of the southern sample were obtained with the CTIO Infrared Imager on the $1.5 \mathrm{~m}$ telescope on three nights ( 2 in October 1989 and 1 in January 1990). All the stars were observed at least once, except star 24, which was too bright for reliable observation. Nearly half the stars were observed more than once. Standard star observations indicate typical uncertainties for the data of $0.03 \mathrm{mag}$, but comparison of multiple measurements of program stars shows slightly greater variation. For stars observed once, the estimated uncertainty in $K$ is $0.04 \mathrm{mag}$, and $0.03 \mathrm{mag}$ or better for the $J-K$ and $H-K$ colors; these uncertainties are independent of magnitude. Color transformations to the standard CIT/CTIO $J H K$ system (Elias et al. 1982) were determined and applied to the data. Uncertainties in the color transformation are not significant, since the program stars lie within the range in color covered by the standards. The results are summarized in Table 2 . Column (7) refers to the number of different nights that the star was observed. The star name indicates both the number of the star and its approximate spectral type. The latter is self-explanatory, except that " $H$ " implies that the star shows $\mathrm{H} \alpha$ in emission.

Eleven members of the southern sample are $M$ giants, and they are markedly brighter than the carbon stars. Their low velocity dispersion $(39 \pm 8 \mathrm{~km} / \mathrm{s})$ suggests membership of the extended disk population (Norris 1987a,b). Five of these stars are listed as detections in the IRAS Point Source Catalogue (Table 3 ). The brightest carbon star, $C^{*} 15$, is not listed in the PSC.

\section{DISCUSSION}

\subsection{Sample Properties}

All but one of the known carbon stars in the Galaxy are evolved stars (see Dearborn et al. 1985). Since the apparent $K$ magnitudes of our sample are in the range $K=9-15 \mathrm{mag}$, and the Galactic latitude $|b|>40^{\circ}$, these carbon stars are all likely to be distant halo giants. Distances can be assigned by fitting their $J-K$ colors to the giant branch of Mould et al. (1984). This method is only approximate and we assign random errors of $30 \%$ in this distance determination. Furthermore, these distances are calculated assuming that Milky Way halo carbon stars populate a metal-poor giant branch. However, recent photometry of carbon stars in the Galactic bulge (Westerlund et al. 1990; Tyson \& Rich 1990) indi- 

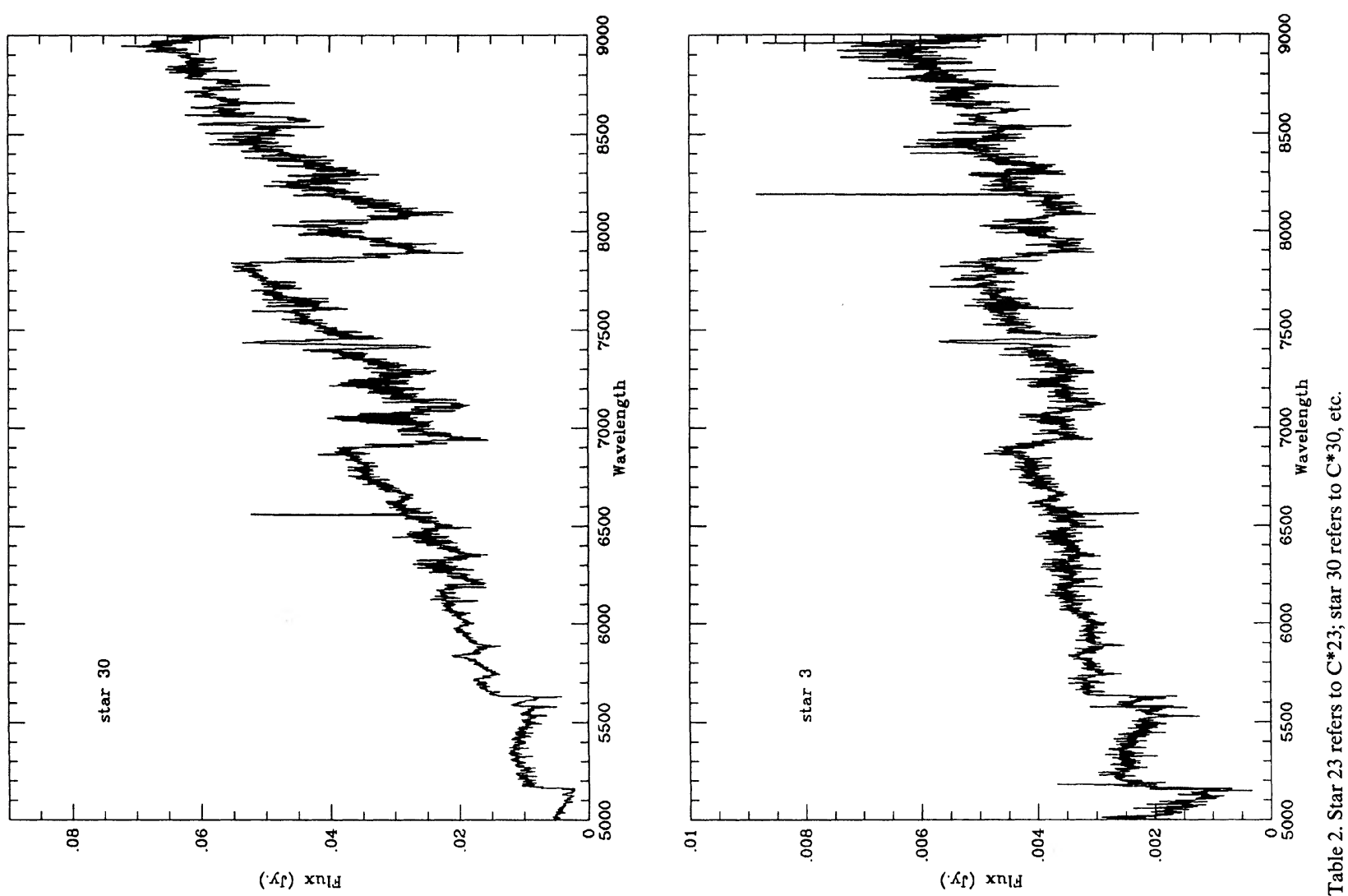

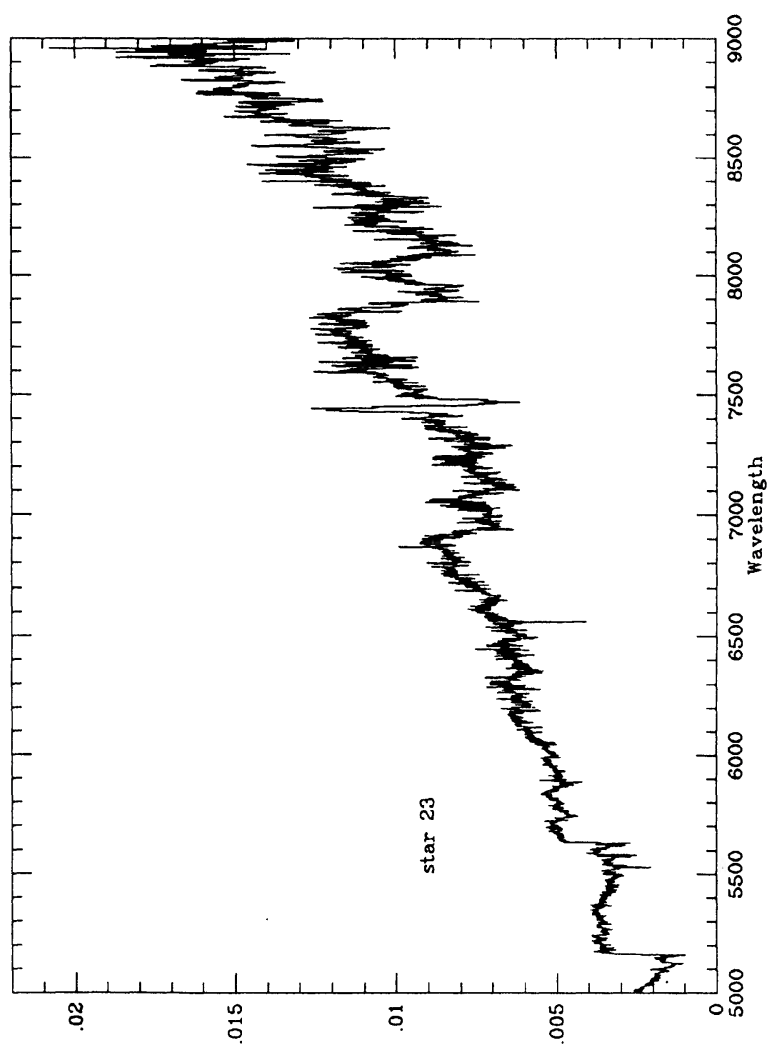

$\left(\mathcal{K}_{f}\right) \times n_{[\mathbb{S}}$

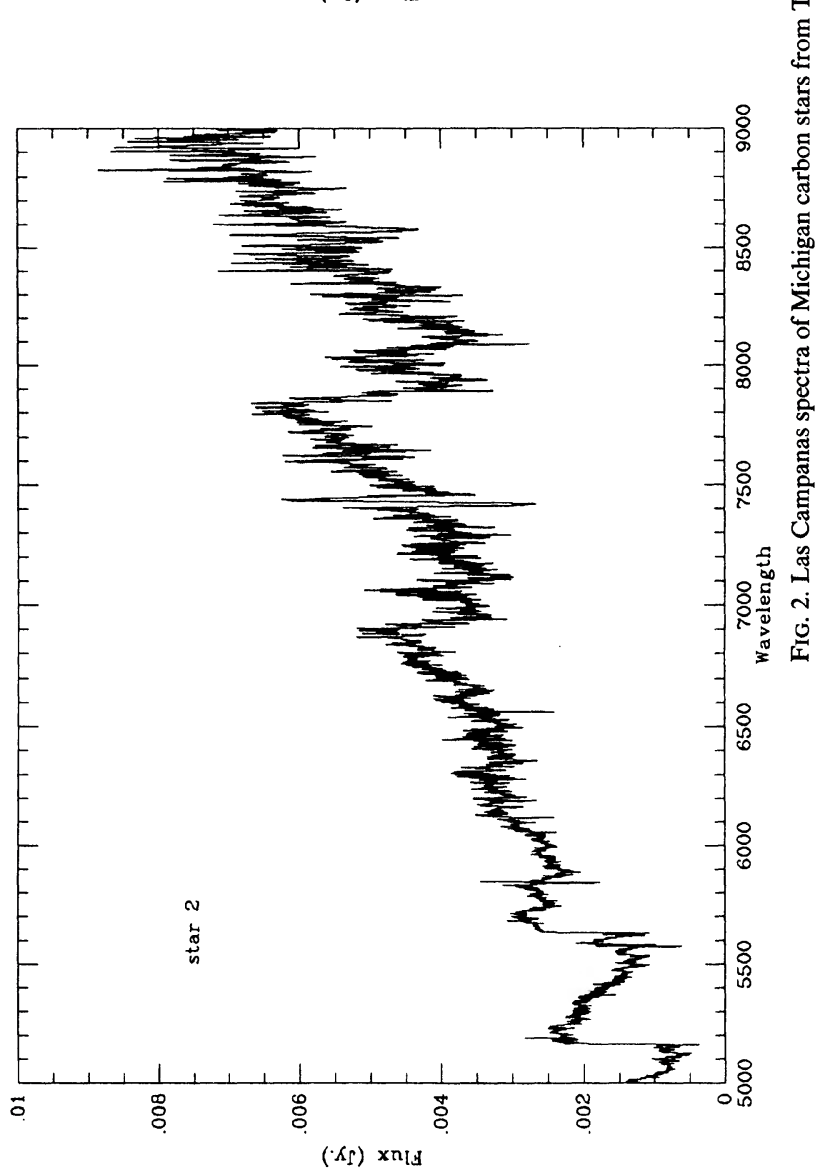




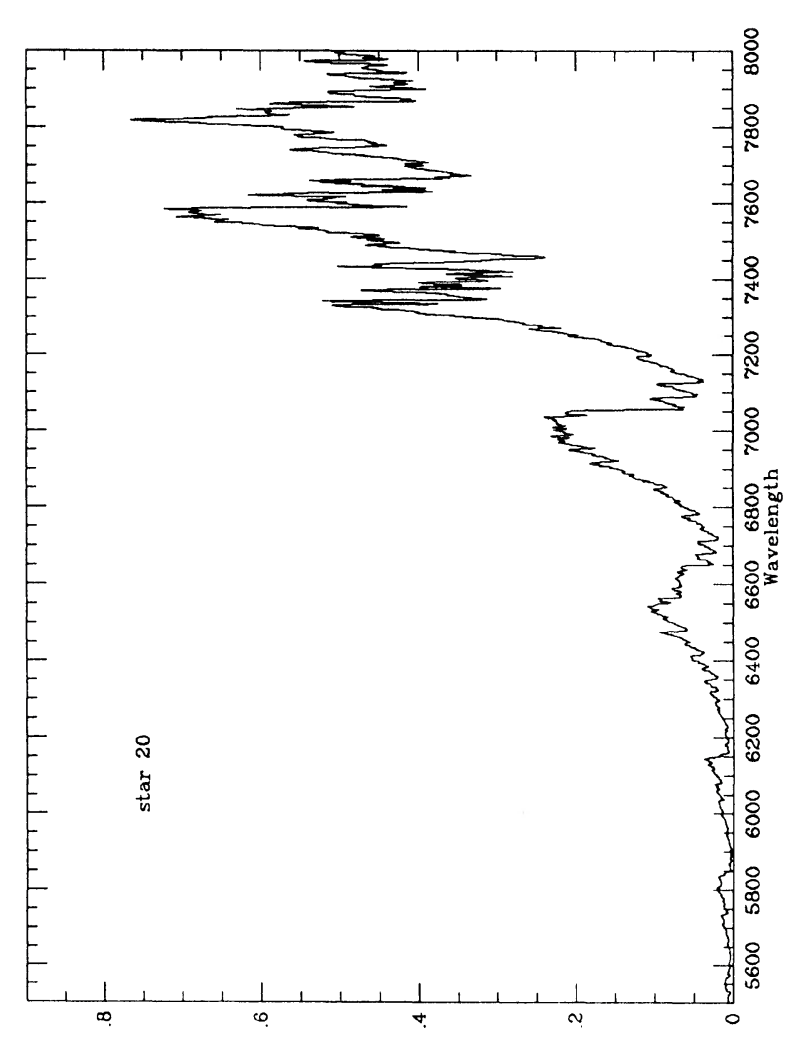

$\left(K_{\Gamma}\right) \times n_{L H}$

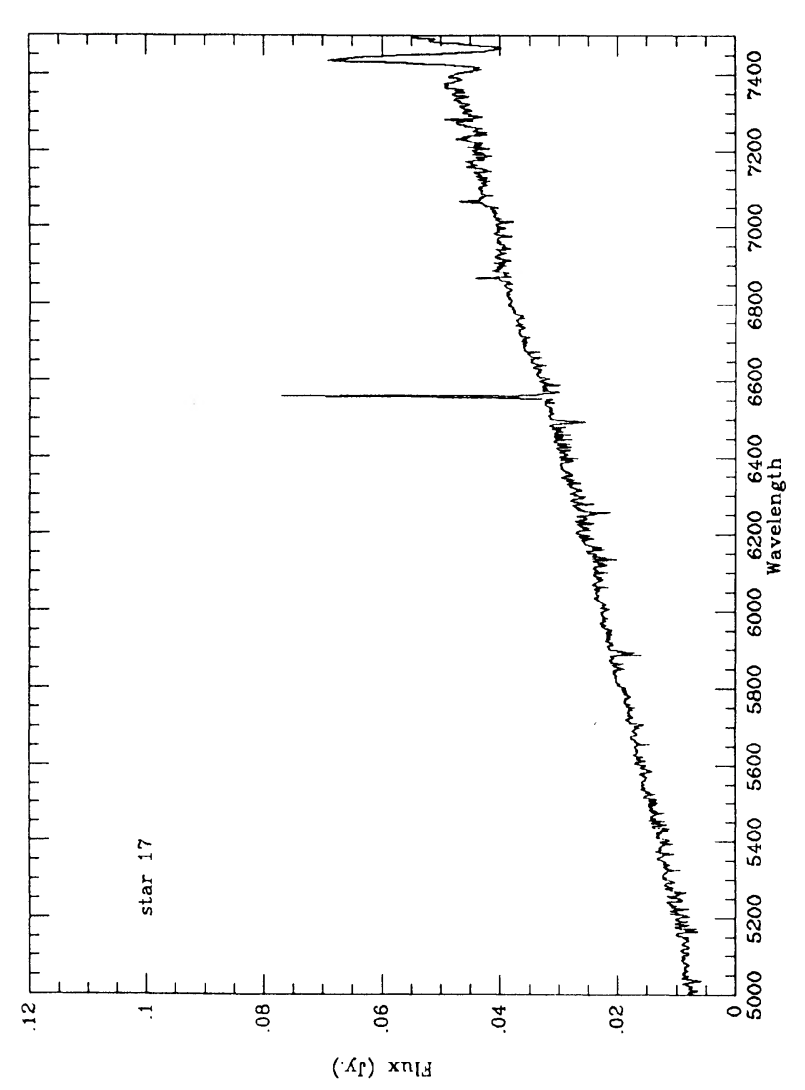

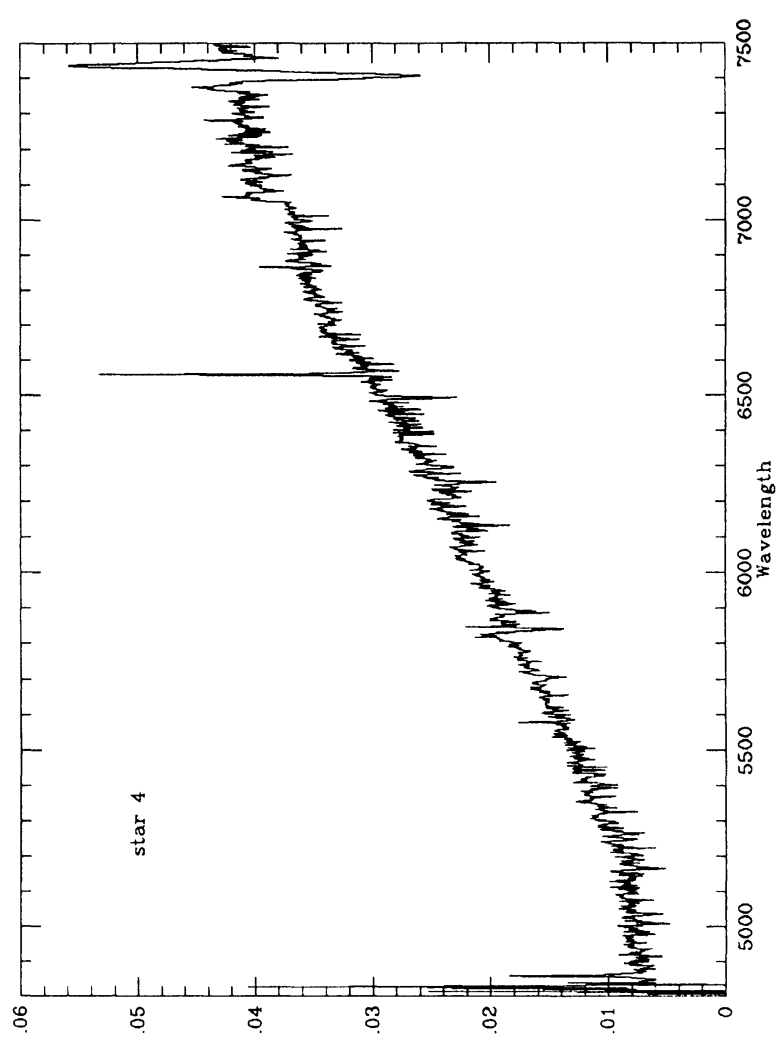

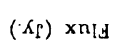

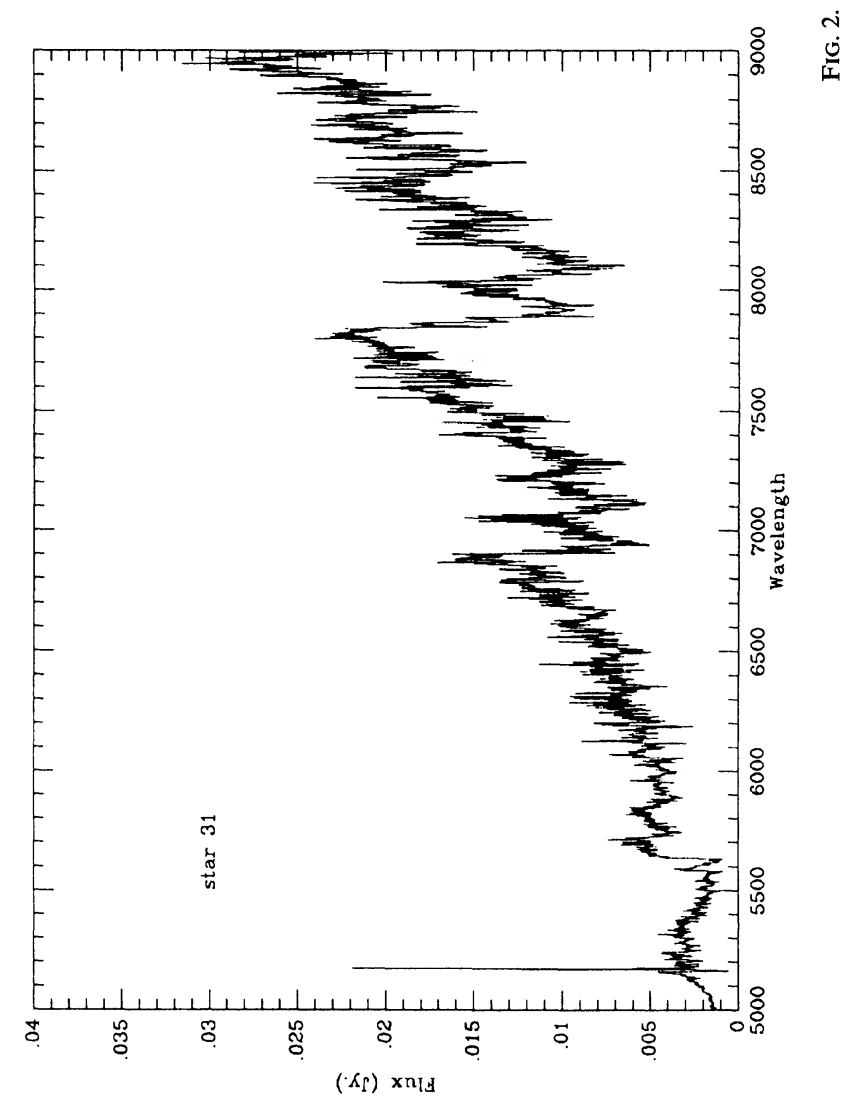


TABLE 2. Photometry and kinematics of the SGP sample.

\begin{tabular}{|c|c|c|c|c|c|c|c|}
\hline $\begin{array}{l}\text { Star } \\
\text { (1) }\end{array}$ & $\begin{array}{l}\text { R.A. } \\
(2)\end{array}$ & $\begin{array}{l}\text { (1950) Dec. } \\
(3)\end{array}$ & $\begin{array}{l}\mathrm{K} \\
(4)\end{array}$ & $\begin{array}{l}\mathrm{J}-\mathrm{K} \\
(5)\end{array}$ & $\begin{array}{l}\mathrm{H}-\mathrm{K} \\
(6)\end{array}$ & $\begin{array}{l}\text { n } \\
(7)\end{array}$ & $\begin{array}{l}\text { Velocity } \\
\text { (8) }\end{array}$ \\
\hline$C^{*} 01$ & $00: 02: 51$ & $+00: 53: 50$ & 10.16 & 0.84 & 0.22 & 2 & 17 \\
\hline $\mathrm{C}^{*} 02$ & $23: 54: 39$ & $+00: 21: 30$ & 12.88 & 0.73 & 0.08 & 2 & -72 \\
\hline $\mathrm{C}^{*} 3$ & 01:08:51 & $-00: 15: 50$ & 12.75 & 0.72 & 0.06 & 1 & -143 \\
\hline $\mathrm{H}^{*} 04$ & $01: 21: 42$ & $+00: 56: 40$ & 10.18 & 0.81 & 0.16 & 1 & \\
\hline$M^{*} 05^{6}$ & $01: 20: 12$ & $+00: 16: 50$ & $8.34^{5}$ & 0.73 & 0.14 & 1 & 4 \\
\hline $\mathrm{C}^{*} 07$ & 01:02:29 & $-05: 56: 10$ & 8.71 & 2.40 & 1.18 & 1 & 21 \\
\hline $\mathrm{C}^{*} 08$ & $02: 28: 46$ & $-02: 56: 20$ & 11.66 & 0.87 & 0.20 & 2 & -72 \\
\hline$C^{*} 09$ & $02: 29: 49$ & $-03: 16: 50$ & 10.69 & 1.12 & 0.32 & 1 & -128 \\
\hline$C^{*} 10$ & $23: 46: 41$ & $+02: 48: 20$ & 12.26 & 0.82 & 0.17 & 2 & -143 \\
\hline$M^{*} 11^{6}$ & $01: 52: 57$ & $+06: 18: 10$ & 10.15 & 0.86 & 0.31 & 2 & 22 \\
\hline$M^{*} 12$ & $23: 55: 20$ & $-09: 14: 10$ & 4.34 & 1.27 & 0.52 & 1 & 79 \\
\hline$C^{*} 13$ & $23: 04: 59$ & $-13: 56: 50$ & 12.70 & 0.83 & 0.18 & 2 & \\
\hline$M^{*} 14^{6}$ & $23: 53: 33$ & $-14: 15: 00$ & 10.35 & 0.85 & 0.33 & 1 & 0 \\
\hline$C^{*} 15$ & $02: 22: 45$ & $-13: 37: 20$ & 6.09 & 1.24 & 0.34 & 3 & -11 \\
\hline$M^{*} 16$ & 03:09:21 & $-11: 56: 40$ & 7.70 & 0.92 & 0.19 & 2 & 23 \\
\hline $\mathrm{H}^{*} 17$ & $22: 37: 21$ & $-17: 38: 30$ & 9.83 & 0.96 & 0.24 & 1 & -156 \\
\hline$M^{*} 18$ & $22: 21: 29$ & $-20: 03: 30$ & 6.49 & 1.10 & 0.37 & 1 & -48 \\
\hline$M^{*} 19$ & $22: 54: 26$ & $-20: 36: 40$ & 3.02 & 1.21 & 0.49 & 1 & -4 \\
\hline$M^{*} 20$ & $23: 10: 48$ & $-15: 35: 30$ & 3.43 & 1.31 & 0.52 & 1 & 51 \\
\hline$M^{*} 21$ & $00: 20: 00$ & $-18: 49: 50$ & 7.01 & 1.08 & 0.34 & 1 & -4 \\
\hline $\mathrm{C}^{*} 22$ & $00: 23: 30$ & $-19: 35: 10$ & 11.64 & 0.90 & 0.32 & 2 & 56 \\
\hline $\mathrm{C}^{*} 23$ & $01: 00: 13$ & $-16: 19: 10$ & 12.51 & 0.62 & 0.06 & 2 & -140 \\
\hline$M^{*} 24$ & 01:32:01 & $-19: 13: 30$ & & & & & -24 \\
\hline $\mathrm{M}^{*} 25$ & $02: 29: 38$ & $-19: 43: 50$ & 3.50 & 1.36 & 0.44 & 2 & 67 \\
\hline$C^{*} 26$ & $22: 13: 43$ & $-14: 51: 20$ & 11.03 & 0.93 & 0.26 & 1 & 0 \\
\hline$M^{*} 27$ & $22: 22: 21$ & $-12: 31: 30$ & 6.87 & 1.13 & 0.29 & 1 & 12 \\
\hline$M^{*} 28$ & $23: 51: 52$ & $2-21: 24: 50$ & 5.71 & 1.20 & 0.31 & 1 & 60 \\
\hline$M^{*} 29$ & $03: 07: 47$ & $-27: 42: 40$ & 3.74 & 1.17 & 0.30 & 2 & 39 \\
\hline$C^{*} 30$ & $02: 07: 40$ & $-02: 12: 00$ & 9.72 & 1.18 & 0.42 & 2 & -131 \\
\hline $\mathrm{C}^{*} 31$ & $02: 17: 49$ & $+00: 56: 50$ & 9.73 & 1.23 & 0.35 & 1 & -138 \\
\hline$M^{*} 32$ & $23: 59: 34$ & $-14: 57: 10$ & & & & & 100 \\
\hline
\end{tabular}

${ }^{3}$ Heliocentric radial velocity in $\mathrm{km} / \mathrm{sec}$

${ }^{5}$ Double star; photometry includes both components. ${ }^{6} \mathrm{M}$ dwarf

cates a redder giant branch. Distances calculated from the average bulge giant branch are on average half those discussed here. Conclusions about the kinematics of the sample, however, are unaffected by which of the two distance scales is adopted. The range in distances is significantly larger in the NGP sample than the SGP one. Both samples, however, have a similar median distance of $28 \mathrm{kpc}$. The most remote carbon star in either sample is CLS 31 located at a distance of $115 \pm 35 \mathrm{kpc}$, assuming the metal-poor distance scale.

TABLE 3. IRAS PSC fluxes.

\begin{tabular}{lrrr}
\hline \hline Star & $12 \mu \mathrm{m}$ & $25 \mu \mathrm{m}$ & $60 \mu \mathrm{m}$ \\
\hline $\mathrm{M}^{*} 12$ & 3.63 & 1.43 & $<0.40$ \\
$\mathrm{M}^{*} 19$ & 13.20 & 5.33 & 0.89 \\
$\mathrm{M}^{*} 24$ & 7.58 & 2.77 & 0.41 \\
$\mathrm{M}^{* 25}$ & 5.48 & 2.49 & $<0.45$ \\
$\mathrm{M}^{*} 32$ & 13.33 & 3.98 & 0.71 \\
\hline \hline
\end{tabular}

Notes to TABLE 3

Fluxes are in Jy. None are detected at $100 \mu \mathrm{m}$.
The ensemble properties of the NGP and SGP carbon star samples are compared in Table 4. The velocity dispersion of the NGP sample is marginally $(2.3 \sigma)$ higher. However, this can be attributed to the significantly larger range in distances sampled by the NGP stars. A more perplexing difference between the two samples lies in their respective distributions of $J-K$ color. Although there is a $3.0 \sigma$ difference in mean color (with star C*07 in the SGP sample excluded-see below) it is perhaps more significant that the reddest star in the NGP sample has $J-K=0.93$ mag whereas the colors of 6 of the 13 SGP stars exceed this value. While we can offer no salient explanation for this difference, it seems likely that it reflects the red continuum color criteria for carbon star candidates which was applied to the southern data.

We also note that one star in the southern sample $\left(\mathrm{C}^{*} 07\right)$ is extremely red. Its color is matched only by the asymptotic giant branch carbon stars in the Magellanic Clouds. If we assume that $M_{K}=-8 \mathrm{mag}$, which is the mean value in the LMC, this star is $22 \mathrm{kpc}$ distant and is certainly a halo star. Unless there is some way of creating intermediate-mass stars like this one in stellar mergers, it is necessary to invoke contamination of the Galactic halo by an intermediate-age population in order to explain stars such as this and $1523+426$ (Mould et al. 1984). The main-sequence progenitors of this population may be the high velocity A stars (Rodgers et al. 1981). There is an additional curiosity in the SGP dataset in that stars $C^{*} 30$ and $C * 31$ are located within 3 degrees of one another and have identical $K$-band magnitudes, $J-K$ colors, and radial velocities. This local surface density is a factor of 20 larger than the global surface density of carbon stars derived earlier, which may indicate that these stars trace some other coherent stellar system buried in the halo.

\subsection{Structure of the Galactic Halo}

In Fig. 3(a) we plot the raw distance distribution of the combined NGP and SGP carbon star samples. The data are binned in rings of width $5 \mathrm{kpc}$. The deficit of carbon stars in the inner bin is to be expected since nearby carbon stars would be rather overexposed on these deep survey plates. To turn these raw counts into a surface density profile, we need to correct for the volume element $\left(d r^{2}\right)$. Figure $3(\mathrm{~b})$ shows the surface density profile that is obtained in this manner. The data are plotted in $r^{1 / 4}$ space so that a de Vaucouleurs profile would be a straight line. The slope of the line gives the effective radius for the $r^{1 / 4}$ law. A least-squares fit to the data return a value of $r_{\mathrm{e}} 6.9 \pm 1.0 \mathrm{kpc}$ and that line is plotted as the solid line in Fig. 3(b). The fit to the data is quite good

TABLE 4. Comparison of NGP and SGP samples.

\begin{tabular}{lcc}
\hline \hline & NGP & SGP \\
& $(n=20)^{*}$ & $(n=12)$ \\
\hline Mean velocity (km/s) & $-35 \pm 23$ & $-68 \pm 20$ \\
Dispersion (km/s) & $106 \pm 12$ & $72 \pm 9$ \\
Distance (kpc) & $25 \pm 26$ & $28 \pm 11$ \\
Latitude (degrees) & $59^{\circ} \pm 13$ & $62^{\circ} \pm 8$ \\
$J-K$ (mag) & $0.68 \pm 0.04$ & $0.93 \pm 0.06$ \\
\hline \hline
\end{tabular}

Notes to TABLE 4

(a) Only the 15 objects in Table 1 used to make $J-K$ for NGP. (b) Star C*07 excluded from $J-K$ in SGP.

(c) Errors in mean velocity, dispersion, and $J-K$ are $1 \sigma$ uncertainties in the mean; errors on distance and latitude represent dispersion in the distribution. 

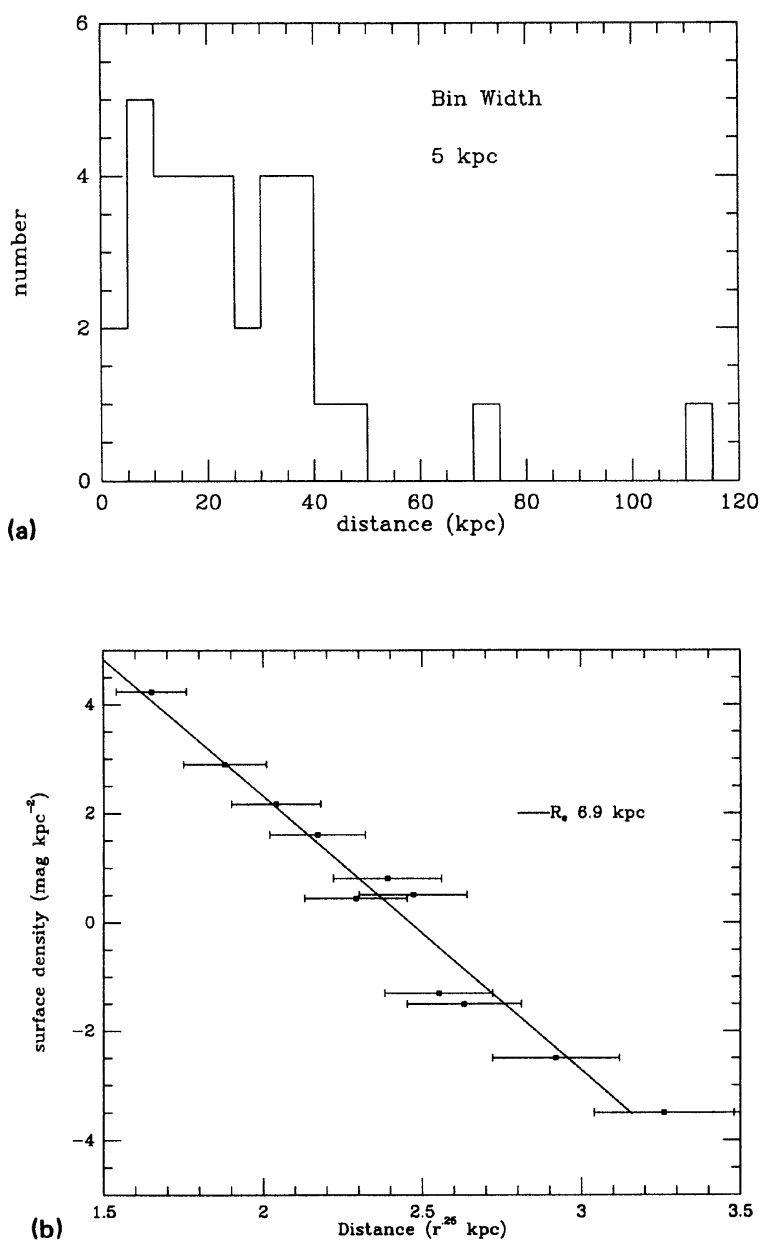

FIG. 3. (a) Histogram of carbon star frequency as a function of distance. (b) Surface density of carbon stars as a function of radius in a spherical halo. In this figure the $x$ axis is in units of $\mathrm{kpc}^{1 / 4}$ and the $y$ axis shows the profile in mag $\mathrm{kpc}^{-2}$.

with $r=0.98$ and $\chi^{2}=0.92$. For the case where the carbon stars are like those in the Galactic bulge, all scales quoted above should be reduced by a factor of 2 . Thus, any determination of the proper value of $r_{\mathrm{e}}$ from this data is dominated by the systematics of choice of distance scale instead of random error.

We also have used the available globular cluster data (e.g., Harris \& Racine 1979) and treated it in the same manner. In Fig. 4 we plot the surface density of globulars in comparison to the carbon stars. At large distances the surface density profiles are in reasonable agreement whereas there are significantly more globulars at small radii. This, of course, indicates a smaller value of $r_{\mathrm{e}}$ for the globulars which is to be expected since the globular cluster distribution may be strongly affected by dynamical friction (e.g., Ostriker 1988) and hence no longer accurately reflects $r_{\mathrm{e}}$ of the halo proper. A fit to the globular cluster data formally yields $r_{\mathrm{e}}=5.0 \pm 0.85 \mathrm{kpc}$ but this value is artificially large since, in order to compare directly to the carbon star data, we do not use data inside of $d=5 \mathrm{kpc}$. From this we conclude that if the carbon star distribution really traces the globular clus-

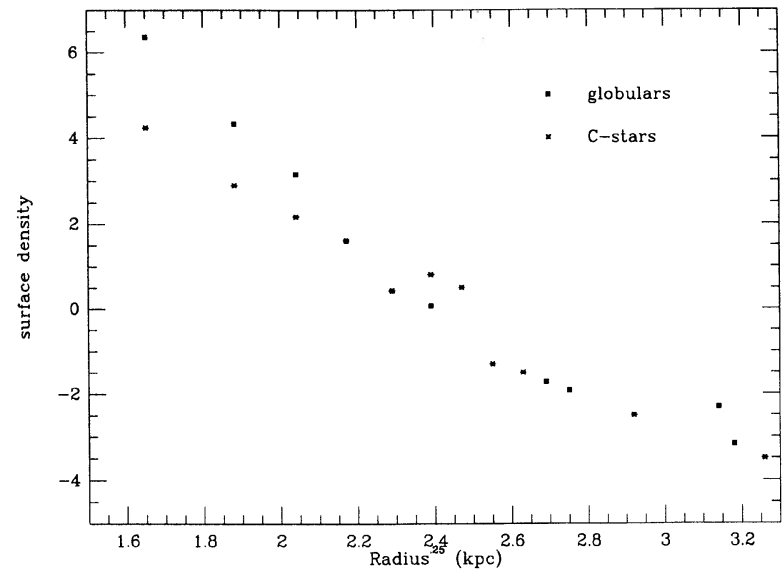

FIG. 4. Comparison of the surface density distribution of globular clusters and carbon stars. The units are as in Fig. 3(b) (i.e., the ordinate is a log scale) and the globular cluster data are binned in an identical manner to the carbon star data.

ter distribution, then a distance scale midway between the metal-poor and metal-rich alternatives is suggested. However, there are sound dynamical reasons to expect, after 1 Hubble time has elapsed, that halo stars and globular clusters would have differing distributions.

The above exercise is not meant to provide a definitive value for $r_{\mathrm{e}}$ since the systematics of our distance scale are rather unknown. The real value in Fig. 3(b) is the demonstration that (1) the Galactic halo is well described by a de Vaucouleurs surface density profile and (2) the actual data formally extend out to $\approx 15 r_{\mathrm{e}}$. This latter point is truly remarkable. Elliptical galaxies, for instance, do not have light profiles which are traceable out to $15 r_{\mathrm{e}}$. Clearly a larger sample of distant tracers is required to test the dimensions of the Galactic halo.

\subsection{Kinematic Properties}

We can examine the kinematics of the sample by assuming a model in which the system has a velocity of $-220 \mathrm{~km} / \mathrm{s}$ in the standard direction relative to the local standard of rest, and the Sun has a velocity of $12 \mathrm{~km} / \mathrm{s}$ in that direction relative to the LSR. The expected mean heliocentric radial velocity in that model is $-49 \mathrm{~km} / \mathrm{s}$ for our sample; the observed velocity is $-48 \pm 17 \mathrm{~km} / \mathrm{s}$. The agreement is excellent.

We can also use the data to constrain the vertical velocity dispersion of this relatively distant set of tracers. Two predictions are available for the velocity dispersion of the sample. There is the cylindrical model of Ratnatunga \& Freeman (1985) in which the velocity ellipsoid of the halo maintains a minor axis pointed at the Galactic poles. This predicts $\sigma=80 \mathrm{~km} / \mathrm{s}$. The alternative model is the spherical model in which the velocity ellipsoid is everywhere elongated radially towards the Galactic center (Pier 1984), which predicts $\sigma=122 \mathrm{~km} / \mathrm{s}$. Our combined sample of carbon stars yields a value of $\sigma=96 \pm 12 \mathrm{~km} / \mathrm{s}$. Moreover, as shown in Fig. 5, 


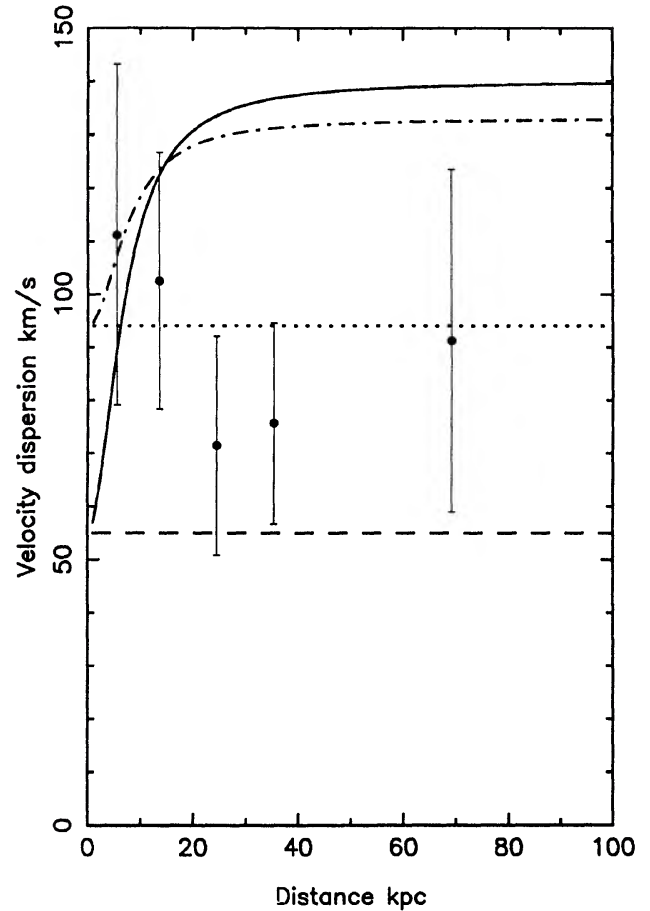

FIG. 5. Velocity dispersion as a function of distance. The curves show the prediction of models at the Galactic poles. Solid curve: the spherical model with the velocity ellipsoid of Hartwick (1983). Dashed line: the cylindrical model of Ratnatunga \& Freeman (1985). Dot-dashed curve: the spherical model with the velocity ellipsoid of Morrison et al. (1990). Dotted line: the cylindrical model with the velocity ellipsoid of Morrison et al. (1990).

the dispersion in this sample is independent of distance above the Galactic plane. Our value is clearly intermediate between the predictions of the two models. If we modify the Ratnatunga and Freeman model, employing the halo velocity ellipsoid of Morrison et al. (1990) in place of that of
Hartwick (1983), we see that the cylindrical model provides a satisfactory fit to the data, but more data are clearly required, especially at distances larger than $40 \mathrm{kpc}$.

\section{SUMMARY}

From objective prism surveys we have formed a small sample of high galactic latitude carbon star candidates. Spectroscopic observations of these candidates have yielded a sample of 32 verified carbon stars which have galactic latitude $\geqslant 40^{\circ}$. Twenty are located at the North Galactic Pole while twelve comprise the southern sample. $J H K$ photometry is used to determine $\mathscr{M}_{\text {bol }}$ from which distances can be derived. Our analysis of this small sample yields the following results:

(1) Some of the carbon stars in the southern sample are quite red and may trace an intermediate-age population in the Galactic halo.

(2) The most distant star in the sample is located at $115 \pm 35 \mathrm{kpc}$.

(3) The velocity dispersion of the combined sample is $96 \pm 12 \mathrm{~km} \mathrm{~s}^{-1}$; a value intermediate between the spherical model of Pier (1984) and the cylindrical model prediction of Ratnatunga \& Freeman (1985).

(4) Within the data, a model in which the velocity dispersion is independent of height above the galactic plane is preferred. This model best fits the data if we adopt the halo velocity ellipsoid proposed by Morrison et al. (1990).

(5) The surface density profile of halo carbon stars is very consistent with a de Vaucouleurs $r^{1 / 4}$ falloff. The data covers $\approx 15$ scale radii which indicates that the halo of our Galaxy may be a rather large structure.

We thank Robert Wagner for preparation of finding charts, Bruce Margon for communication of results prior to publication and Heather Morrison for helpful discussions. We thank the personnel of Palomar, Cerro Tololo, and Las Campanas Observatories for their assistance with the observations. Infrared astronomy at Palomar is supported by a grant from the National Science Foundation.
Aaronson, M. 1983, ApJL, 266, L11

Boothroyd, A., and Sackmann, I. 1988, ApJ, 328, 653

Cohen, J., Frogel, J., and Persson, S. E. 1981, ApJ, 249, 481

Cook, K., and Aaronson, M. 1989, AJ, 97, 927

Dearborn, D., Liebert, J., Aaronson, M., Dahn, C., Harrington, R., Mould,

J., and Greenstein, J. 1985, ApJ, 300, 314

Elias, J., Frogel, J., Matthews, K., and Neugebauer, G. 1982, AJ, 87, 1029

Hartwick, F. D. A. 1983, Mem. della Soc. Astr. Italiana, 54, 51

Green, P., and Margon, B. 1990, preprint

Green, P., and Margon, N. 1991, in preparation

Hartwick, F. D. A., and Cowley, A. 1985, AJ, 90, 2244

Kinman, T. 1991, preprint

Iben 1975, ApJ, 196, 525

Iben, I., and Renzini, A. 1983, ARA\&A, 21, 271

Lattanzio, J. 1986, ApJ, 311, 708

MacAlpine, G., and Lewis, D. 1978, ApJS, 36, 587

McClure, R. 1984, ApJL, 280, L31; 1989, private communication

Morrison, H., Flynn, C., and Freeman, K. 1990, AJ, 100, 1191

Mould, J., and Da Costa, G. 1988, in Progress and Opportunities in South-

ern Hemisphere Astronomy, ASP Conference Series, edited by V. Blanco

\section{REFERENCES}

and M. Phillips (Bringham Young University Press, Provo, UT), p. 197 Mould, J., Schneider, D., Gordon, G., Aaronson, M., and Liebert, J. 1984, PASP, 97, 131

Norris, J. 1987a, AJ, 93, 616

Norris, J. 1987b, ApJL, 314, L39

Oke, J. B., and Gunn, J. 1982, PASP, 94, 586

Ostriker, J. 1988, The Harlow Shapley Symposium, edited by J. Grindlay (Reidel, Dordrecht), p. 271

Pesch, P., and Sanduleak, N. 1988, ApJS, 66, 387

Pier, J. 1984, ApJ, 281, 260

Ratnatunga, K., and Freeman, K. 1985, ApJ, 291, 260

Reid, I. N., Tinney, C., and Mould, J. 1990, ApJ, 348, 98

Rodgers, A., and Paltoglou, G. 1984, ApJL, 283, L5

Rodgers, A., Harding, P., and Sadler, E. 1981, ApJ, 244, 912

Salzer, U. 1987, Ph. D. thesis, University of Michigan

Schechter, P. 1988, A Guide to the Modular Spectrograph (OCIW)

Stone, R., and Baldwin, J. 1983, MNRAS, 204, 347

Tyson, N., and Rich R. M. 1990, preprint

Westerlund, N., Lequeux, J., Azzopardi, M., and Rebeirot, E. 1990, Observatoire de Marseille, Prepublication Nos. 106 and 107 